\title{
Technohomeopathy, the environment friendly application of homeopathy in technology - a review
}

\begin{abstract}
Homeopathic drug aggregates achieve nanodimension at higher potency. For the first time we have utilized this nanoparticle aspect in various technical applications, giving birth to the science of Technohomeopathy. We have shown that thermo- and photovoltage generation in selected systems gets enhanced in presence of the homeopathic medicine, triturated Zincum oxydatum, thus making itself an agent for efficient solar energy conversion. Similarly, incorporation of the homeopathic medicines, e.g. Cuprum metallicum (triturated $\mathrm{Cu}$ ) and Cobaltum metallicum (triturated $\mathrm{Co}$ ), in a polymer PVDF, which is commonly used as filler material for capacitors in electronic industry, enhances the electrical properties. The effects in all these cases increase with increase in potency. These applications of homeopathic medicines, which are cost effective and easily available, are in the true sense environment friendly.
\end{abstract}

Keywords: technohomeopathy, homeopathic medicine, nanoparticle aspect, solar energy conversion, electrical properties, polymer
Volume 3 Issue 5 - 2018

\author{
Papiya Nandy \\ Centre for Interdisciplinary Research and Education, India
}

Correspondence: Papiya Nandy, Center for Interdisciplinary Research \& Education, 404 B Jodhpur Park, Kolkata-700 068, India,Tel 9830021785,Email pnandy00@gmail.com

Received: February 18, 2017 | Published: September 20, 2018

\section{Introduction}

Homeopathy is a time-tested, empirical system of healing that has been used universally for more than 200 years, because of its negligible side effects, low cost, easy availability and easier applicability. However, The molecular basis of the medicinal action of the drug, at very high dilution, (one part in 10400 parts), has been a subject of controversy for a long time, leading to various complicated theories which are yet to be proved and confirmed. In order to explain why dilution increases the potency of homeopathic medicines, we presumed that during the succession process used in preparing high potency homeopathic medicine, perhaps a large amount of mechanical energy is transferred to the system, causing reduction in the size of the original aggregated drug particles to nano-dimension.1 Using two different kinds of homeopathic medicines of hydrophobic and hydrophilic nature, we have shown that the liposomal membrane anisotropy gets modulated with the potency, thereby indicating facilitated permeation of drugs through the membrane.2-4 Later, experimental evidence of formation of nanoparticle at higher potency was reported by us, which was supported by several other research groups, and thus the nanoparticle aspect of homeopathic medicine was firmly established.5-8 For the first time we have taken this property of nanoparticle formation of homeopathic medicine one step further, by utilizing them in the following technological applications.

Homoeopathic medicine Zincum oxydatum used as an agent for enhancement of thermo- and photo- voltage generation

Harnessing solar energy using inexpensive techniques is an important challenge, and newer concepts are being developed for solar to electrical energy conversion. In search of newer materials for efficient conversion of solar energy into electrical energy, several nanomaterials have been engineered.9 The invention of nanoparticles, which possess the unique chemical and physical properties with size and shape variations, has a strong impact in photovoltaic technology as they can facilitate the conversion process of solar energy to electrical energy. Several nanoparticles have been used to convert solar energy in our laboratory. While searching for novel suitable materials, the idea of utilization of the nanoparticle aspect of the homeopathic medicine Zincum oxydatum, (triturated $\mathrm{ZnO}$ ) was developed by us.10 Field emission scanning electron microscopy image shows the morphology of the medicine (Figure 1).

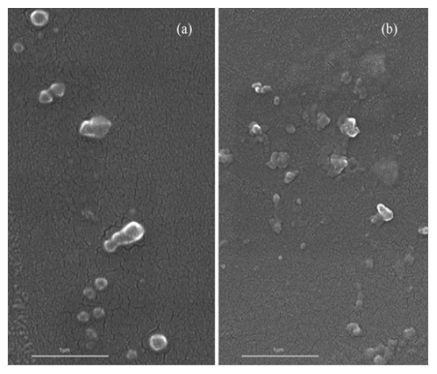

Figure I FFESEM image of zincum oxydatum (a) 6C potency and (b) $30 \mathrm{C}$ potency. Measured average values of sizes of Zincum oxydatum nano particles for $6 \mathrm{C}$ and $30 \mathrm{C}$ potency are $83.1 \mathrm{~nm}$ and $1 \mathrm{I} .0 \mathrm{Inm}$ respectively.

A specially devised electrochemical cell was used for the study of thermo- and photovoltage generation as shown in Figure 2.

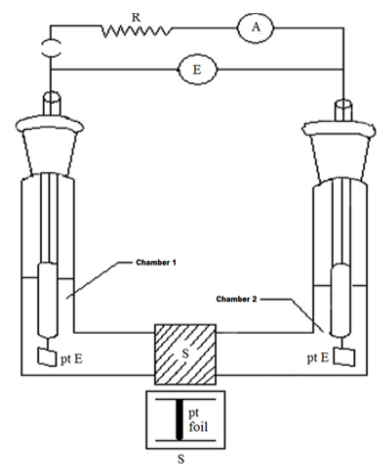

Figure 2 The electrochemical cell used for the experiments for solar energy conversion (thermo and photo voltage generation)." 


\section{Thermo-voltage generation}

Using triturated $\mathrm{ZnO}$, we have been able to generate thermo-voltage.10 Maximum voltage generated was found to increase with potency of the medicine (Figure 3 ). The efficiency of the cell with the medicine at potency $30 \mathrm{C}$ at $40^{\circ} \mathrm{C}$ was calculated from the current-voltage curve (Figure 4 ) and the value of efficiency is $\sim 0.39 \%$.

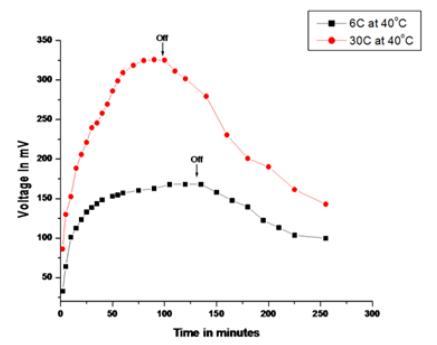

Figure 3 Growth and decay curve of $\mathrm{Voc}$ (thermo-voltage) generation using triturated $\mathrm{ZnO}$ at potencies $6 \mathrm{C}$ and $30 \mathrm{C} .{ }^{10}$

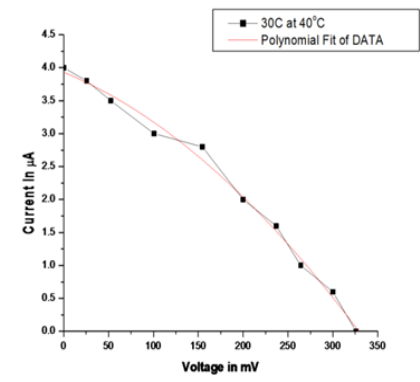

Figure 4 Current vs. voltage characteristic curves for the Zincum oxydatum of potency $30 \mathrm{C}$ at $40 \circ \mathrm{C}$ using platinum barrier. 10

\section{Enhancement of efficiency of solar energy converter} utilizing the broad solar spectrum range

To ensure the performance of the electrochemical cell over a broad solar spectrum range, two dyes, Azure $\mathrm{C}$ and Rose Bengal, having absorption bands in two different spectral regions, $545 \mathrm{~nm}$ and $610 \mathrm{~nm}$ respectively, were chosen in order to overcome the band absorption limits of each dye and were combined together. These dyes were used in conjunction with triturated $\mathrm{ZnO}$. Our result shows that photo induced voltage with significant efficiency is generated when triturated $\mathrm{ZnO}$ is used in combination with these two different photosensitive organic dyes. The photovoltage generation in EC cell has been observed using Azure $\mathrm{C}$ and Rose Bengal solution and triturated zinc oxide (Figure 5). The I-V characteristic of the cell was studied (Figure 6) and the energy conversion efficiency $(\eta \%)$ of the cell has been calculated for all four cases (Table 1). In all cases, photo induced voltage generation upon illumination started rising with time and reached a maximum saturation value and remained constant at that value. When the light was cut off the voltage decreased slowly and the storage duration for $30 \mathrm{C}$ was more than $4 \mathrm{hrs}$ to reach the initial value. Also at this potency, higher efficiency of PEC cell was obtained. The photovoltage cycle was reproducible upon repeat illumination.

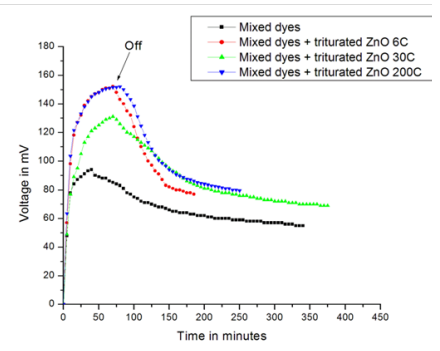

Figure 5 Growth and decay curve of Voc (photovoltage) generation for mixed Azure C, Rose Bengal and triturated zinc oxide of potency $6 \mathrm{C}, 30 \mathrm{C}$ and 200C.

Table I The characteristics of electrochemical cell using mixed Azure C, Rose Bengal and triturated $\mathrm{ZnO}$ of potency $6 \mathrm{C}$, 30C and $200 \mathrm{C}$

\begin{tabular}{|c|c|c|c|c|c|}
\hline Sample used & $\begin{array}{l}\text { Peak value of } \\
\text { photovoltage }(\mathrm{mV})\end{array}$ & $\begin{array}{l}\text { Short circuit } \\
\text { current }(\mu \mathrm{A})\end{array}$ & $\begin{array}{l}\text { One cycle duration } \\
\text { (minutes) }\end{array}$ & Fill factor (FF) & $\begin{array}{l}\text { Energy conversion } \\
\text { efficiency }(n \%)\end{array}$ \\
\hline Only mixed dye & 95 & 5 & 310 & 0.38 & 0.15 \\
\hline Mixed dye $+\mathrm{ZnO} 6 \mathrm{C}$ & 150 & 6 & 248 & 0.52 & 0.39 \\
\hline Mixed dye + ZnO 30C & 134 & 9.5 & 375 & 0.4 & 0.43 \\
\hline Mixed dye + ZnO 200C & 152 & 6 & 255 & 0.5 & 0.35 \\
\hline
\end{tabular}

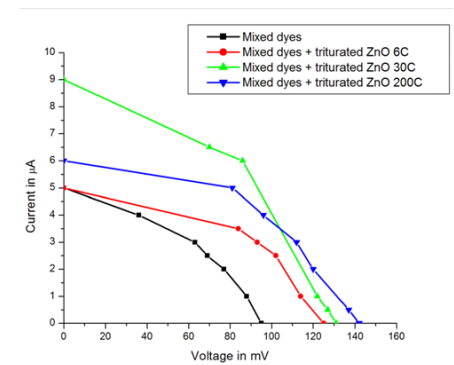

Figure 6 Current versus voltage characteristic curves for mixed Azure C, Rose Bengal and triturated zinc oxide of potency $6 \mathrm{C}, 30 \mathrm{C}$ and $200 \mathrm{C}$.

The energy conversion efficiency of the cell using three different potencies $6 \mathrm{C}, 30 \mathrm{C}$ and $200 \mathrm{C}$ of the medicine with same concentration of the two dyes $\left(0.5 \times 10^{-5} \mathrm{M}\right)$ in all cases are $0.39 \%, 0.43 \%$ and $0.35 \%$ respectively. The efficiency is only $0.15 \%$ for the mixed dye under similar conditions (Table 1). ${ }^{11}$ In order to understand how triturated $\mathrm{ZnO}$ tuned the photoelectric properties of the dye molecules, the absorption spectra of the mixed dye in presence of triturated zinc oxide at different potencies had been studied (Figure 7). Azure $\mathrm{C}$ and Rose bengal have their characteristic bands at $545 \mathrm{~nm}$ and $610 \mathrm{~nm}$ respectively. With addition of the triturated $\mathrm{ZnO}$, the characteristic peaks of the dye remain unchanged but absorbance increased. Our experimental results on photovoltage generation using triturated $\mathrm{ZnO}$ show presence of nanoparticles (Figure 1) of almost spherical shape and particle sizes within 5-7 nm.,10 Due to adsorption of dye molecule on the surface of triturated $\mathrm{ZnO}$ nanoparticles, the absorption efficiency of the dye molecule increased. ${ }^{12}$ There is also a high possibility of forming exciplex between these two dyes at excited state which could not participate in the process of conversion of solar energy. Addition of triturated $\mathrm{ZnO}$ also plays a vital role here. As the 
dyes got adsorbed on the surface of these nanoparticles, they could participate now actively in the conversion process and the problem of spectral loss was reduced by better exploitation of the incident photon. The increase in efficiency of triturated $\mathrm{ZnO}$ at $30 \mathrm{C}$ from that of $6 \mathrm{C}$ can be explained from the fact that with increasing potency the particle size decreases, leading to increased surface to volume ratio. ${ }^{5}$ However at $200 \mathrm{C}$, the concentration of nanoparticle is too low to participate in the conversion process resulting in lower efficiency than those at $6 \mathrm{C}$ and 30C. Our study of photovoltage generation in a dye-sensitized photo-electrochemical cell using Azure C, Rose Bengal and triturated $\mathrm{ZnO}$ at three different potencies shows that with addition of triturated zinc oxide in mixed solution of Azure $\mathrm{C}$ and Rose Bengal system, the magnitude of photovoltage, storage duration and energy conversion efficiency gets enhanced significantly and has maximum value at $30 \mathrm{C}$ potency. Spectral studies reveal that with addition of the nanoparticle, the characteristics of the dye solution remains unchanged while only overall optical density of the system increases indicating that there is no chemical reaction between the dyes and the triturated zinc oxide nanoparticle.

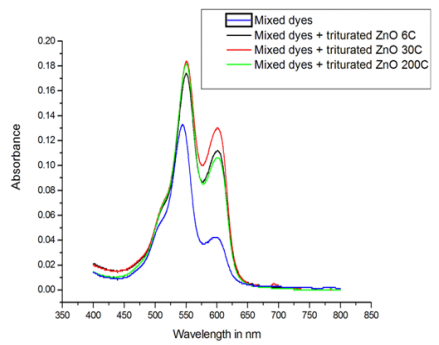

Figure 7 Absorption spectra of mixed azure C, Rose Bengal and triturated zinc oxide system with concentration of mixed dyes solution $0.85 \mu \mathrm{M}$ and triturated zinc oxide of potency $6 \mathrm{C}, 30 \mathrm{C}$ and $200 \mathrm{C}$.

Improvisation of electrical properties of PVDF-HFP: use of homeopathic medicines, novel metallic nanoparticles

Poly (Vinylidene fluoride) (PVDF) and its copolymers like polyvinylidene fluoride-hexa-fluoropropylene (PVDF-HFP) are in great demand for their versatile and unique properties like flexibility, low processing temperature, low dielectric constant, high dielectric breakdown field etc., making them potential candidates for a broad range of applications in electronic industry such as dielectric filler for condensers. ${ }^{13-15}$ In order to improve the capacitive performance of the polymer material, incorporation of metal nanoparticles in the polymer matrix has received great attention. The uniform and homogeneous distribution of the nanoparticles allows good interaction between them and the polymer matrix material and enhances the electrical properties of the host material. ${ }^{16}$ In this endeavour, a great deal of effort had been devoted to develop PVDF-HFP composites by incorporating different metallic nanoparticles within the matrix. The effective dielectric permittivities of these metal nanoparticle doped polymer composites are higher than that of the host polymer matrix. They also show enhancement of conductivity and decrement of tangent loss making them potential candidates as good capacitors and electric energy storage devices. ${ }^{17-22}$

Here we have chosen two homeopathic medicines, viz., Cuprum metallicum (triturated copper) and Cobaltum metallicum (triturated cobalt), both very novel and unique metallic fillers, which are nontoxic, inexpensive and are easily available. As homeopathic medicines, they affect the membrane permeability and their nanoparticle aspect has been proved experimentally ${ }^{2,5}$ These novel nanoparticles were incorporated in the polymer PVDF-HFP in dimethyl-sulfoxoide solution and films were prepared by simple solution casting technique. These nanocomposites of PVDF-HFP/triturated $\mathrm{Cu}(\mathrm{CuPC})$ and $\mathrm{PVDF} /$ triturated Co (CoPC) were studied by FTIR, FESEM and dielectric analysis. ${ }^{23}$ The presence of $\alpha$ and $\beta$ phases, formation of spherulites, enhancement of dielectric constant and conductivity of the nanocomposite films were observed by changing the potency of triturated $\mathrm{Co}$ and triturated $\mathrm{Cu}$ and the observed values were compared with that of the pure PVDF-HFP film. The presence of $\alpha-$ and $\beta-$ phases and spherulitic crystal structure of PVDF-HFP of these nanocomposite films have been detected by Fourier Transform Infrared Spectroscopy (FTIR) study (Figure 8). The transition of phases between $\alpha$ and $\beta$ is activated by the incorporation of metallic nanoparticles in the polymer matrix. This provides the nanocomposites higher mobile charge carriers which participate in the interfacial polarization.

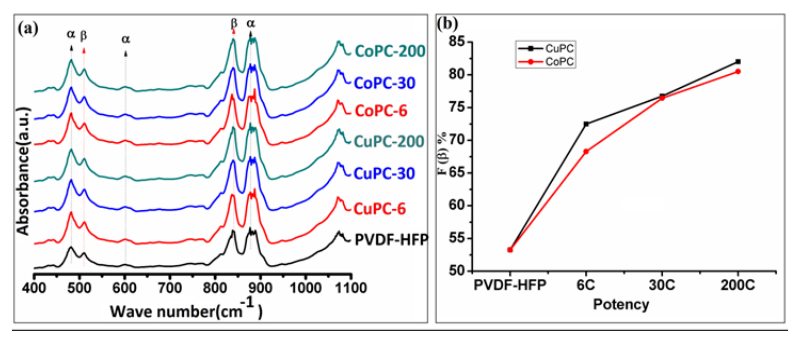

Figure 8 (a) Fourier transform infrared spectra of CuPC and CoPC for all potency $(b)$ Increase of $F(\beta) \%$ with increase in potency.

\section{FTIR analysis}

Fourier Transform Infrared Spectroscopy (FTIR) study of these nanocomposite films in the selected range of $400-1,100 \mathrm{~cm}^{-1}$ detected presence of $\alpha$ - and $\beta$-phases, Figure 8a. The FTIR spectra of CuPC and $\mathrm{CoPC}$ nanocomposite film show characteristic absorbance bands at 484,606 and $878 \mathrm{~cm}^{-1}$ corresponding to the $\alpha$-phase and around 510 and $840 \mathrm{~cm}^{-1}$ corresponding to the $\beta$-phase. The spectra indicate that there are no phase shifts or chemical interactions between the metal nanoparticles and the polymer film, but the intensity of $\alpha-$ and $\beta$-phases changes with the concentration of doping material. We observe in Figure $8 \mathrm{~b}$ that with increase in potency, the $\beta$-phase peaks have grown significantly compared to those of PVDF-HFP. Using Beer-Lambert law, which relates absorbance with concentration of the absorbing species, the fraction of $\beta$-phase, $F_{\beta}$, present in the (crystalline regions of) films was calculated using the formula,

$$
F(\beta)=\frac{A \beta}{1.26 A \alpha+A \beta}
$$

Where, $A_{\alpha}$ and $A_{\beta}$ are the absorption fractions of $\alpha$ and $\beta$ - phases at $484 \mathrm{~cm}^{-1}$ and at $840 \mathrm{~cm}^{-1}$ respectively.

Undoped PVDF had $53 \% \beta$-phase that increased with the addition of the different potency of the nanoparticles. The enhancement in $\beta$-phase was maximum ( $82.04 \%$ ) for $\mathrm{TCu}$ nanoparticles at $200 \mathrm{C}$ potency among all the composites. All composite films showed a constant increasing trend with increase in potency.

\section{FESEM analysis}

Figure 9a-9f show the morphology and microstructure of all composite films doped with $\mathrm{TCu}$ and TCo nanoparticles of 6C, 30C and 200C potencies respectively. Figure 9a \& 9d are evidence of large 
number of densely packed agglomerated particles embedded in the polymer matrix, whereas Figure 9b, c, e \& f show that the particles are more scattered, well separated and also homogeneously distributed maintaining an intermolecular distance.
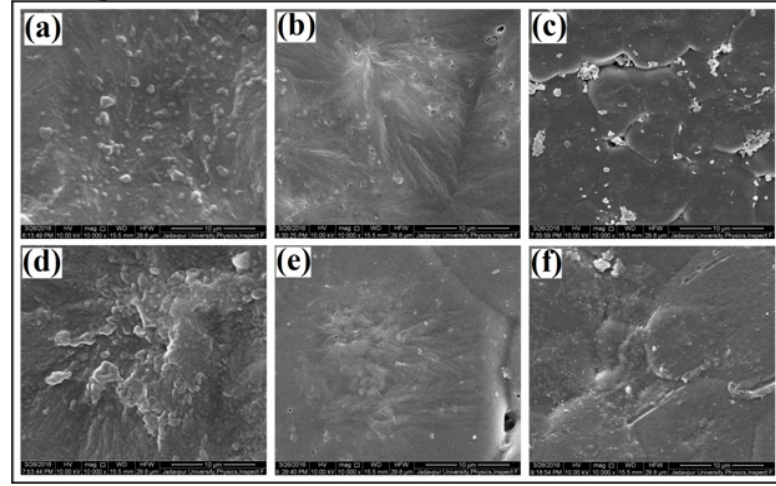

Figure 9 Field-emission scanning electron micrograph (FESEM) of (a) CuPC6,(b) CuPC-30, (c) CuPC-200, (d) CoPC-6, (e) CoPC-30 and (f) CoPC-200 films.

\section{Dielectric measurements}

The variations of dielectric constant of all $\mathrm{CuPC}$ and $\mathrm{CoPC}$ film with frequency are shown in Figure 10a \& 10b respectively. Throughout the whole frequency range, dielectric constant has substantially higher value in case of all $\mathrm{CuPC}$ and CoPC films compared to the pure polymer film. As there are two different conducting media present here (e.g. PVDF-HFP and TCu or TCo), this enhancement may be explained by MWS (Maxwell-Wagner-Sillars) interfacial polarization effect which appears in heterogeneous medium consisting of different phases with different permittivity and conductivity due to accumulation of the charges at the interfaces. ${ }^{24}$ The interfacial polarization is associated with the entrapment or accumulation of free charges generated in the cores at the interfaces between the cores and the matrix. At lower frequency the easy orientation of dipoles as well as MWS interfacial polarization contribute this $(\sim) 4$ times enhancement. As the frequency is increased further, dipole response is restricted and the dielectric constant has a saturation tendency. In this case, the internal individual dipoles contribute to the dielectric

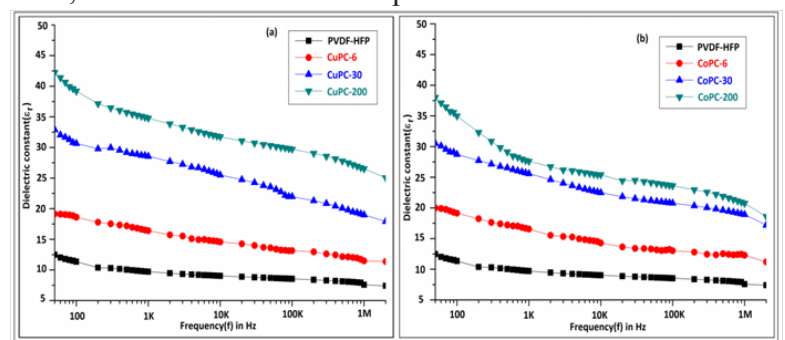

Figure 10 Frequency dependent dielectric constant $\left(\varepsilon_{\mathrm{r}}\right)$ of (a) CuPC and (b) CoPC for all potency. constant which is ideally the electronic polarization effect.

\section{A.C. conductivity}

The variation of ac conductivity with frequency for all $\mathrm{CuPC}$ and CoPC film is shown in Figure 11a \& 11b, respectively. It shows ac conductivity increases exponentially with frequency for both the nanocomposite films.

At higher frequency range, rapid increase of conductivity with increasing frequency is referred to electronic polarization effect. This increase in conductivity with frequency also arises due to the presence of free ions in the polymer matrix that may increase the mobility of the ions which finds an easy path to move and hence increase the electrical conductivity. The value of ac conductivity is higher for all nanocomposite films than the pure polymer film. For lower potencies of these films, the particles are in bulk form and agglomerated which is embedded in the polymer matrix. So the interfacial area per unit volume decreases while the interparticle distance decreases. This decreases the average polarization associated with the particles resulting in the further decrement of dielectric constant and ac conductivity as well as increment of tangent loss. This phenomenon is also clearly observed from their microstructures (Figure 9).

We conclude that incorporation of homeopathic nano particles in PVDF-HFP leads to

a. Gradual increase in electroactive $\beta$-phase compared to PVDF-HFP (Figure 8).

b. As the potency of the medicine is increased, better interaction is observed on polymer matrix and thereby found homogeneously distributed particles (Figure 9).

c. The dielectric constant of all nanocomposites decreases with increase in frequency for all concentrations of metal nanoparticles (Figure 10). This value is higher for higher dilutions and highest for CuPC-200 HNPs concentration (Figure10).

d. The AC conductivity increases with frequency for all nanocomposite films due to the presence of mobile metal ions in the polymer composites (Figure 11).

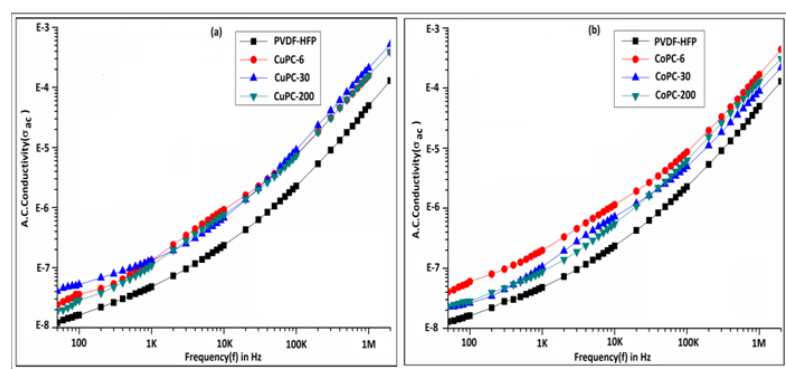

Figure II Frequency dependent ac conductivity $\left(\sigma_{\text {a.c }}\right)$ of (a) CuPC and (b) CoPC for all potency.

We have shown that the incorporation leads to strong interfacial interaction between the HNPs and the polymer resulting in enhanced dielectric constant of the thin films. The observed variation of the dielectric properties of the thin films depends on the surface, size and extent of agglomeration of the HNPs in the polymer matrix. Thus, pure polymer film which has comparatively low dielectric constant can be modified into materials with enhanced dielectric constant by making a composite with triturated metal nanoparticles, which are nontoxic, eco-friendly and easily available in the nano form. As a dielectric material, these nanocomposite films can then be a promising candidate for the fabrication of high charge- storing multilayer capacitors and can be of great use in electronic industry. From these experiments we conclude that homeopathic medicines as nanoparticles can enhance as candidate for the fabrication of high charge-storing multilayer capacitors and can be of great use in electronic industry. Further study of this aspect of homeopathy, which we term as Technohomeopathy, is in progress. 


\section{Acknowledgements}

The work has been done in technical collaboration with the Central Council for Research in Homeopathy, Department of AYUSH, and New Delhi. Equipment facilities are obtained from different departments of Jadavpur University. Drugs were gifts from the manufacturer, Hahnemann Publishing Company, Kolkata, India. All the data presented here are from experiments performed by the author's group. The unpublished results are taken from the communicated papers. Stimulating discussion with Dr. DS Bhar is thankfully acknowledged. The author acknowledges, most happily, the help received from the students and fellow co-workers.

\section{Conflict of interest}

The author declares there is no conflict of interest.

\section{References}

1. Nandy P. A review of basic research on homeopathy from a physicists's point of view. Indian J Research in Homeopathy. 2015;9(3):141-151.

2. Nandy P, Bhandary S, Das S, et al. Nanoparticles and membrane anisotropy. Homoeopathy.2011;100(3):194-197.

3. Ghosh S, Chakraborty M, Das S, et al. Effect of different potencies of nanomedicine Cuprum metallicum on membrane fluidity - A biophysical study. Am J Homeopath Med. 2014;107(4):16-169.

4. Bhandary S, Das S, Basu R, et al. Effect of Aconitum napelles on liposomal microviscosity. Int J Emerg Technol Sci Eng. 2011;3:1-5.

5. Kar S, Bandyopadhyay P, Chakraborty S, et al. Derivation of an empirical relation between the size of the nanoparticle and the potency of homeopathic medicines. Intl J High Dilution Res. 2015;14(4):2-7.

6. Chikramane PS, Kalita D, Suresh AK, et al. Why extreme dilutions reach non-zero asymptotes: A nanoparticulate hypothesis based on froth flotation. Langmuir. 2012;28(4):15864-15875.

7. Chikramane PS, Suresh AK, Bellare JR, et al. Extreme homeopathic dilutions retain starting materials: A nanoparticulate perspective. Homoeopathy. 2010;99(4):231-242.

8. Upadhyay RP, Nayak C. Homoeopathy emerging as nanomedicine. Int J High Dilution Res. 2011;10:299-310.

9. Kamat PV. Meeting the Clean Energy Demand: Nanostructure Architectures for Solar Energy Conversion. J Phys Chem C. 2007;111(7):2834-2860.

10. Bandyopadhyay P, Nandy P, Basu R, et al. Effect of dilution on thermovoltage generation using homeopathic nanomedicine Zincum oxydatum. Int J Innovative Res in Sci and Eng. 2010:3:226-230.

11. Bandyopadhyay P, Basu R, Das S, et al. Enhancement of quantum efficiency of a dye sensitized electrochemical cell by using triturated zinc oxide mixed with two organic dyes, azure c and rose Bengal, 2016.

12. Bandyopadhyay P, Chakraborty S, Basu R, et al. Efficiency of a dye- sensitized photoelectrochemical device using thionine and triturated zinc oxide at different potency. Energy Sources, Part-A: Recov, Utilization and Environ effects.2016;32(13):1833-1839.

13. Martins P, Costa CM, Benelmekki M, et al. On the origin of the electroactive poly(vinylidene fluoride) $\beta$-phase nucleation by ferrite nanoparticles via surface electrostatic interactions. Cryst Eng Comm. 2012;14(8):2807-2811.

14. Martins P, Lopes AC, Lanceros-Mendez S. Electroactive phases of poly (vinylidene fluoride): determination, processing and applications, Prog. Polym Sci. 2013;39:683-706.

15. Nalwa HS. Ferroelectric polymers: chemistry, physics, and applications. New York, Marcel Dekker, 1995.

16. Li Y, Huang $\mathrm{X}, \mathrm{Hu} \mathrm{Z}$, et al. Large dielectric constant and high thermal conductivity in poly(vinylidene fluoride)/ barium titanate/ silicon carbide three-phase nanocomposites. Appl Mater Interfaces, 2011;3(11):4396-4403.

17. Thakur P, Kool A, Bagchi B, et al. Effect of in situ synthesized $\mathrm{Fe}_{2} \mathrm{O}_{3}$ and $\mathrm{Co}_{3} \mathrm{O}_{4}$ nanoparticles on electroactive $\beta$ phase crystallization and dielectric properties of poly(vinylidene fluoride) thin films hys. Chem Chem Phys. 2015:17:1368-1378.

18. Dang ZM, Wang L, Yin Y. Giant dielectric permittivities in functionalized carbon-nanotube/electroactive-polymer nanocomposites. Adv Mater. 2007;19(6):852-857.

19. He F, Lau S, Chan HL, et al. High dielectric permittivity and low percolation threshold in nanocomposites based on poly(vinylidene fluoride) and exfoliated graphite nanoplates. Adv Mater. 2009;21(6):710-715.

20. Thakur P, Kool A, Bagchi B, et al. Enhancement of $\beta$ phase crystallization and dielectric behavior of kaolinite/halloysite modified poly(vinylidene fluoride) thin films. Appl Clay Sci. 2014;99:149159.

21. Paul BK, Kar S, Bandyopadhyay P, et al. Significant enhancement of dielectric and conducting properties of electroactive polymer polyvinylidene fluoride films: an innovative use of Ferrum metallicum at different concentrations. Indian J Res Homeopath. 2016;10(1):5257.

22. Paul BK, Das S, Basu R, et al. Effect of dilution of Ferrum metallicum and zincum oxidatum, homeopathic nanomedicines on the dielectric properties of poly(PVDF-HFP) film. Int $J$ High Dilution Res. 2016;15(1):10-17.

23. Gayen L, Paul BK, Roy D, et al. Improvisation of electrical properties of PVDF-HFP: use of novel metallic nanoparticles. Journal of Materials Science: Materials in Electronics. 2017;28(19): 14798-14808.

24. Rogti F, Ferhat M. Maxwell-Wagner polarization and interfacial charge at the multi-layers of thermoplastic polymers. $J$ Electrost. 2014;72(1):91-97. 